\title{
Adoecimento e finitude: considerações sobre a abordagem interdisciplinar no Centro de Tratamento Intensivo oncológico
}

\author{
Illness and finitude: considerations about the interdisciplinary \\ approach in the oncological Intensive Care Unit
}

Luciana da Silva Alcantara ${ }^{1}$

Joana Lezan Sant'Anna ${ }^{1}$

Maria da Glória Nascimento de Souza ${ }^{1}$

Abstract Being afflicted with cancer involves a high degree of invasive and mutilating treatment, which has repercussions on the lives of individuals and harks back to the recollection of pain, limitations and fear of death, which requires the participation of the family or person responsible for the patient during treatment. Attention to the relatives or persons responsible for the patient is a very important evaluation issue of care in health institutions, as an integral aspect of the process of humanization of assistance, that requires the training of professionals to identify and meet their needs. Given the above, this study seeks to address some considerations regarding the interdisciplinary work of assisting the patients' families in the Adult Intensive Care Unit of Cancer Hospital I, as an efficient proposal with respect to attention to such clinical information requirements, as well as social, medical and/or psychological guidance.

Key words Finitude, Interdisciplinarity, Family, Humanization
Resumo O adoecimento por câncer, que envolve sobremaneira tratamento invasivo e mutilador, traz repercussões na vida dos sujeitos, remontando a um imaginário de associação à dor, limitações e receio da morte, o que demanda a participação do familiar ou responsável pelo paciente durante o tratamento. O atendimento aos familiares ou responsáveis pelo paciente é um importante conteúdo da avaliação do cuidado nas instituições de saúde, enquanto aspecto integrante do processo de humanização da assistência e que requer dos profissionais a capacitação para identificar e atender suas necessidades. Diante do exposto, o presente estudo tem como objetivo trazer à tona algumas considerações sobre o trabalho interdisciplinar no acolhimento à família dos pacientes do Centro de Tratamento Intensivo adulto do Hospital do Câncer I, enquanto proposta eficiente no que tange ao atendimento de tais necessidades de informação clínica, orientação e suporte social, clínico elou psicológico.

Palavras-chave Finitude, Interdisciplinaridade, Família, Humanização
${ }^{1}$ Instituto Nacional de Câncer José Alencar Gomes da Silva (INCA). Praça da Cruz Vermelha 23, Centro. 20230-130 Rio de Janeiro RJ.luerj2004@yahoo.com.br 


\section{Considerações iniciais}

\section{Características do adoecimento por câncer no Brasil: o tênue limite entre o tratamento curativo e o cuidado paliativo}

Acompanhando a tendência mundial, notamse, no Brasil ${ }^{1}$, processos de transição que têm produzido significativas mudanças no perfil das enfermidades que acometem a população, observando-se, a partir dos anos 1960, que as doenças infecciosas e parasitárias deixaram de ser a principal causa de morte, sendo substituídas pelas doenças do aparelho circulatório e pelas neoplasias. As estimativas para o ano de 2012, também válidas para 2013, apontam a ocorrência de aproximadamente 518.510 casos novos de câncer, incluindo os de pele não melanoma, reforçando a magnitude do problema do câncer no país ${ }^{1}$. Esta progressiva ascensão da incidência e da mortalidade por doenças crônico-degenerativas, conhecida como transição epidemiológica, tem como principal fator o envelhecimento da população, fruto do intenso processo de urbanização e das ações de promoção e recuperação da saúde.

Considerando a atenção oncológica, podemos destacar que o perfil epidemiológico do câncer vem suscitando, mundialmente, a preocupação governamental, conquistando espaço nas agendas políticas e técnicas das esferas de governo. No contexto brasileiro, no que tange à prevenção e controle do câncer, o Instituto Nacional de Câncer José Alencar Gomes da Silva (INCA) desenvolve ações, campanhas e programas em âmbito nacional, em consonância com a Política Nacional de Atenção Oncológica do Ministério da Saúde (Portaria GM/MS 2.439/2005)².

Ao considerar a diversidade regional, a Política Nacional de Atenção Oncológica (PNAO) reconhece a integralidade como diretriz para organização da linha de cuidados em atenção oncológica, tendo como premissas a hierarquização e a regionalização dos serviços em todas as unidades federadas, com vistas a garantir condições de acesso da população aos cuidados em oncologia e ao diagnóstico precoce na rede básica, através da qualificação e treinamento dos profissionais do nível primário de saúde. $\mathrm{O}$ acesso aos serviços de atenção básica no Brasil, entretanto, ainda apresenta sérias limitações. As desigualdades regionais e sociais no acesso e na qualidade da atenção em saúde representam para os usuários do sistema, em muitos casos, a impossibilidade do acesso a um diagnóstico precoce e da chance de realizar um tratamento curativo, e não paliativo.
A realidade oncológica aponta para a chegada aos centros de referência de alta complexidade com doença avançada ao diagnóstico, face aos aspectos supracitados. Receber o diagnóstico de uma doença como o câncer provoca transformações importantes na vida das pessoas, com implicações sociais, emocionais e físicas, além de agravar dificuldades referentes à falta de recursos e de vínculos familiares, situações potencialmente geradoras de conflito. Contudo, se por um lado o diagnóstico do câncer remonta a um imaginário de associação à dor, às limitações físicas e ao receio da dependência e da morte, por outro, a revelação do diagnóstico ao paciente permite a ele e à sua família o acionamento de estratégias para lidar, conforme o possível, com os efeitos causados pela doença e seu tratamento.

Por ser uma doença que pressupõe tratamento de longa duração, os profissionais do INCA atuam na identificação das condições de vida e dos determinantes sociais que interferem no processo saúde-doença, para, a partir daí, mobilizar os usuários e a família no sentido do referenciamento aos recursos internos e externos à unidade de saúde. Cabe considerarmos a qualidade da atenção em saúde não apenas condicionada ao acesso ao tratamento, mas também à garantia de acesso aos procedimentos (exames, cirurgias, entre outros) e à efetivação do sistema de contrarreferência aos serviços externos, de modo a propiciar o alcance da atenção integral à saúde da população $0^{3,4}$.

Esta realidade impõe aos profissionais de saúde a necessidade de aprimoramento profissional, na perspectiva de uma intervenção que fortaleça o Sistema Único de Saúde (SUS) e mecanismos que contribuam para a efetivação do acesso dos usuários às Políticas Públicas, bem como a garantia de continuidade do tratamento. $\mathrm{Na}$ atuação em oncologia, a perspectiva de uma atenção integral ao usuário é fundamental.

Com a proposta de reforço da atenção integral à saúde foi lançada, em 2003, a Política Nacional de Humanização $(\mathrm{PNH})^{4}$, que busca colocar em prática os princípios do SUS no cotidiano dos serviços de saúde, sugerindo mudanças nos modos de gerir e cuidar. Vinculada à Secretaria de Atenção à Saúde do Ministério da Saúde, no Departamento de Ações Programáticas e Estratégicas (DAPES), a PNH conta com um núcleo técnico sediado em Brasília/DF e equipes regionais de apoiadores que se articulam às secretarias estaduais e municipais de saúde. Desta articulação são construídos, de forma compartilhada, planos de ação para promover e disseminar inovações em saúde. 
A Política Nacional de Humanização do SUS, em seus dispositivos, vem ao encontro das estratégias no atendimento à pessoa com câncer, promovendo uma abordagem que considera a perspectiva do cuidado em saúde e que viabiliza a efetivação dos direitos dos usuários, possibilitando a realização do Controle Social 5 . O cuidado em saúde não se trata apenas de um nível de atenção do sistema de saúde, mas corresponde a uma ação integral que tem significados e sentidos voltados para compreensão de saúde como o direito de ser, respeitando as relações de etnia, gênero e raça. Equivale a enxergar além da patologia, percebendo necessidades específicas. Pressupõe garantir acesso às outras práticas terapêuticas, permitindo ao usuário decidir acerca da melhor tecnologia médica a ser utilizada por ele 6 .

Contudo, é pertinente enfatizar que a construção de uma política pública de tal dimensão não se sustenta apenas em seu conteúdo formal: depende de ações e conexões com o coletivo. A humanização ${ }^{7}$, enquanto política transversal que atualiza um conjunto de princípios e diretrizes por meio de ações, práticas em saúde e instâncias, só se efetiva a partir da construção coletiva. Desse modo, na qualificação do SUS, a humanização não é apenas mais um programa a ser aplicado aos serviços de saúde, mas uma política que opera de forma transversal em toda a rede.

O conhecimento sobre a doença e sua evolução permite o estabelecimento de prioridades em relação ao atendimento prestado. Inúmeras razões contribuem para que o atendimento hospitalar seja prestado de forma paliativa, e não preventiva, entre elas o fato de os aspectos preventivos e educacionais ainda não terem alcançado efetiva prioridade governamental. A demanda por tratamento altamente especializado, como é o caso dos Centros de Tratamento Intensivo (CTI), pressupõe investimento em recursos humanos e financeiros, além de equipamentos e exames diagnósticos.

Logo, diante do exposto, este breve estudo apresenta uma reflexão acerca da experiência de intervenção interdisciplinar no atendimento aos familiares/responsáveis pelos pacientes internados no CTI adulto do Hospital do Câncer I (HC I), uma das unidades integrantes do INCA, nos contornos da Política Nacional de Atenção ao Paciente Crítico, da Política Nacional de Humanização e na promoção da educação em saúde.

\section{Contextualização do atendimento em situações críticas/potencialmente críticas}

\section{Considerações sobre a Política Nacional de Atenção ao Paciente Crítico e o incentivo à atuação interdisciplinar}

Não é novidade que a expectativa de vida da população brasileira aumentou nas últimas décadas e que a população idosa é cada vez maior. Em consequência, aumentaram os problemas de saúde relacionados à idade avançada e a concentração das internações hospitalares nessa faixa etária. Aliado ao aumento da expectativa de vida, um dos principais desafios atuais dos países em desenvolvimento é o de definir e implementar estratégias efetivas para a prevenção e controle das doenças e agravos não transmissíveis (DANT). Estes agravos passaram a predominar nas estatísticas de saúde, constituindo problemas emergentes nos países em desenvolvimento e nos grupos sociais mais pobres, representando gastos em tratamento ambulatorial, internações hospitalares e reabilitação pelo Sistema Único de Saúde ${ }^{1}$.

$\mathrm{O}$ adequado atendimento a pessoas em situações críticas/potencialmente críticas de saúde está no contexto da atual política de saúde do país, pois elas demandam alto volume, intensidade e complexidade de cuidados, objetivando a detecção precoce de deterioração clínica e conduta terapêutica imediata ${ }^{8}$. Trata-se da Política Nacional de Atenção ao Paciente Crítico (Portaria MS 1.071, de 04 de julho de 2005) ${ }^{9}$, submetida à Consulta Pública em 07 de julho de 2005.

O CTI ou UTI (Unidade de Terapia Intensiva $)^{10}$, por ser considerado uma unidade que possui tecnologia avançada e sofisticada, bem como recursos humanos qualificados para a resolução dos problemas de pacientes críticos com eficiência e eficácia, cria nos trabalhadores a expectativa de eliminarem o desconforto, a dor e o sofrimento dos pacientes, expectativa essa muitas vezes frustrada, apesar da tecnologia e recursos empregados. Tal centro de atendimento tecnologicamente diferenciado se posiciona historicamente como unidade fechada e de acesso restrito. A restrição atinge não apenas o universo social, que não pertencente diretamente ao cotidiano da prática hospitalar se encontra circunstancialmente envolvido com esta unidade, mas também as próprias estruturas assistenciais de saúde, tanto em nível hospitalar quanto extra-hospitalar'.

Com o propósito de ampliar a influência assistencial destas unidades tradicionalmente fechadas e restritivas sobre as demais estruturas/uni- 
dades dos hospitais, a Política Nacional de Atenção ao Paciente Crítico trouxe como inovação a proposta de ampliação de conceitos, objetivando mudanças qualificadoras a serem implementadas. Destaca a interdisciplinaridade enquanto prática necessária ao atendimento integral das necessidades do paciente crítico/potencialmente crítico, compreendendo minimamente a participação partilhada de profissionais da área médica, de enfermagem, psicologia, nutrição, terapia ocupacional, fisioterapia, fonoaudiologia e assistência social, ultrapassando a tradicional preocupação técnica/tecnológica, nos contornos da humanização na saúde.

Aliás, no que tange à humanização, é importante ressaltar o desafio que ela propõe aos profissionais da saúde: o de repensar sua postura profissional. Torna imprescindível a criação de um espaço interdisciplinar para a partilha, a convivência e o estreitamento do vínculo afetivo. Trata-se da humanização sob a ótica da integralidade do sujeito, gerando vínculo afetivo, reflexão intra e interpessoal, desencadeando ações, tais como o desenvolvimento de elos significativos, os quais tornam as pessoas e as situações preciosas e portadoras de valores éticos ${ }^{11}$.

Diante do exposto e tendo em vista a proposta do presente estudo, importa destacar que, para muitos, o CTI é o local onde se trava a "batalha" entre a vida e a morte. Para os pacientes que estão lúcidos, além da dor, estão reservados o tubo, as sondas, os cateteres, a luz incidindo no olho, o barulho dos alarmes, o vozerio dos profissionais, o frio, a nudez, a solidão e o medo da morte ${ }^{12}$.

O local da morte foi paulatinamente transferido do lar para o hospital ${ }^{13}$. Trata-se da morte limpa, longe dos olhos, ilusoriamente sem sofrimento e sem a presença da família. Contudo, indagações de cunho objetivo e subjetivo trazem à tona questionamentos. Para a equipe e a família é o momento de refletir sobre a própria finitude. A partir dessa compreensão, encontramos na proposta interdisciplinar a possibilidade de comunicação efetiva e o estabelecimento de elos entre os profissionais de saúde, os pacientes e seus familiares, enquanto componente capaz de agregar ações pautadas na qualidade do cuidado em saúde.

\section{Discussão}

\section{Grupo de atendimento aos familiares/responsáveis pelos pacientes do CTI adulto do HC I/INCA: o trabalho interdisciplinar na atenção oncológica}

Os trabalhadores do hospital encontram-se divididos em dois grupos: um primeiro formado pelos que lidam diretamente com o doente, sendo este último o objeto de trabalho, bem como a doença e o processo de adoecimento. Nele estão incluídos médicos, enfermeiros, nutricionistas, assistentes sociais, fisioterapeutas, psicólogos, entre outros; já o segundo grupo é formado pelos profissionais que proporcionam sustentação ao trabalho dos primeiros, exercendo função administrativa ou de suporte, nas áreas de manutenção, lavanderia, limpeza, transporte, etc. ${ }^{14}$.

Os profissionais que lidam diretamente com o paciente devem partir do pressuposto de que os instrumentos de trabalho no hospital contemporâneo não são, apenas, as suas instalações e equipamentos, mas o saber dos seus profissionais de saúde categorizados. Concordamos com a concepção ${ }^{15}$ que vem se destacando no campo da Saúde Pública: a de que o avanço na qualidade dos serviços e das ações de saúde, as preventivas ou de promoção, só é possível a partir da participação efetiva da população, que por sua vez tem a dimensão dos seus problemas e possíveis soluções que atendam às suas necessidades.

Nesse sentido, o atendimento, em sua essência, exige dos profissionais a interlocução com a equipe de saúde, no sentido de disseminar a quebra da discriminação e da consequente culpabilização das famílias pelas situações em que se encontram, considerando-as num contexto social mais amplo. Conforme a Carta dos Direitos dos Usuários da Saúde ${ }^{16}$, é assegurado ao usuário o direito a atendimento humanizado, acolhedor e livre de qualquer discriminação, respeitando a sua pessoa, valores e direitos.

Convém destacar que as demandas que chegam aos profissionais atravessam as fronteiras das questões relativas à Política de Saúde: estão atreladas à assistência, exigindo conhecimento da rede por parte do profissional, compreendendo a saúde como um estado físico, mental e social, e não somente como a ausência de doenças. $\mathrm{O}$ hospital, enquanto espaço organizado para atender aos cuidados necessários ao paciente, ocupa na sociedade ocidental um lugar central e quase naturalizado no que concerne à prestação de serviços direcionados à saúde, à doença e à morte ${ }^{17}$. 
Desta forma, precisa estabelecer uma rotina, diferente da que estamos habituados em nosso cotidiano, que vise garantir o andamento do tratamento durante o período de hospitalização.

A realização de um grupo interdisciplinar, por sua vez, abre espaço para um trabalho que depende essencialmente da articulação entre os diferentes saberes, trazendo como ganho o elo entre a família do paciente e os profissionais de saúde. Concordamos que cabe a quem está no front do cuidado acolher as demandas específicas e concretas dos usuários e dos familiares e direcionar um olhar e uma escuta que estabeleçam mediações do geral ao particular ${ }^{18}$.

Atualmente o Instituto Nacional de Câncer José Alencar Gomes da Silva (INCA), órgão integrante do Ministério da Saúde, encontra-se dividido em cinco unidades hospitalares: Hospital do Câncer (HC) I, II, III e IV, e o Centro de Transplante de Medula Óssea (CEMO); o HC I é a maior unidade hospitalar do INCA, atendendo os pacientes portadores de câncer através do SUS.

No CTI adulto do Hospital do Câncer I existem 10 leitos e são internados pacientes oncológicos com intercorrências potencialmente reversíveis. Ocasionalmente são internadas crianças e/ou adolescentes, seja por falta de vaga na unidade infantil ou por se tratarem de pacientes com a compleição física de um adulto.

Nas primeiras horas de internação a expectativa por bons resultados aos cuidados intensivos toma conta da equipe e da família, entretanto nem sempre isso acontece. A condição do paciente pode sofrer complicação apesar dos esforços, não respondendo à proposta terapêutica e com piora progressiva. Pacientes em curva de melhora são acometidos por intercorrências no curso da internação, descortinando a doença neoplásica em franca atividade e sem possibilidade de recuperação. A equipe se sente impotente e o familiar sem esperança. Como lidar com tais circunstâncias?

Desse modo, a perspectiva de um grupo interdisciplinar dedicado ao acolhimento dos usuários partiu da iniciativa dos profissionais envolvidos no atendimento em enfermaria, convictos da necessidade de construção de um elo com a equipe que atua no CTI, para que, desse modo, pudesse ocorrer a aproximação com os familiares/responsáveis dos pacientes internados no CTI. Tal movimento exigiu planejamento cuidadoso da atividade e acompanhamento dos objetivos almejados, considerando que a interdisciplinaridade, ao significar reciprocidade e mutualidade, exige relações sociais horizontais, diferente do posto no modelo assistencial de saúde tradicional, hegemônico ${ }^{19}$.

Contudo, é importante destacarmos, à luz dos estudos vigentes, que a interdisciplinaridade não anula a disciplinaridade, nem tampouco significa a justaposição de saberes. Antes de tudo, suscita consciência dos limites e das potencialidades de cada campo de saber em direção a um fazer coletivo, envolvendo recortes no conjunto do conhecimento. A questão é que um recorte deve ser sempre visto como tal, não substituindo o todo. A interdisciplinaridade no campo da Saúde Pública alcança cada vez mais um status de necessidade, diante da complexidade que o problema da Saúde impõe ${ }^{20}$.

Houve a necessidade, na prática, de estabelecermos relações sustentadas na troca entre as disciplinas e na horizontalização do fazer profissional para, assim, podermos ouvir e refletir sobre o que o outro diz, construindo e reconstruindo novas práticas. Compreendemos, então, esta atividade como espaço para refletir, acolher e coletivizar situações vivenciadas durante o processo de internação hospitalar, publicizar as informações necessárias a uma efetiva qualidade da assistência e contribuir para a promoção da saúde.

A atividade de grupo totaliza onze encontros, com duração média de uma hora, em ambiente fechado, localizado próximo à entrada do CTI, com o objetivo de facilitar o acesso dos participantes. Coordenam a atividade uma assistente social, uma psicóloga, uma enfermeira, uma médica e uma fisioterapeuta. Contamos até o momento com a presença de vinte e nove familiares/responsáveis, dentre eles cinco homens e vinte e quatro mulheres. A diferença significativa da quantidade de membros de cada gênero tem possíveis explicações na maior disponibilidade das mulheres para participação de atividades de grupo que envolvem a fala e na tendência histórica de constituição das mulheres enquanto cuidadoras dos familiares adoentados.

Tendo em vista a dinâmica hospitalar, nos deparamos com o desafio de propiciar um espaço de acolhimento, reflexão e de socialização de informações necessárias aos familiares/responsáveis dos pacientes internados no CTI do HC I/ INCA e que agregasse os profissionais supracitados em torno de um objetivo comum: o de estimular a aproximação entre a equipe de saúde e as famílias atendidas. Nesse sentido, no grupo, destacamos como ganho para a intervenção a preocupação com o acolhimento do usuário e de suas necessidades, de modo que na equipe interdisci- 
plinar os familiares dispõem de acesso aos profissionais, com uma assistência mais resolutiva, que resulta por sua vez em encaminhamentos mais apropriados, evitando desgastes no relacionamento estabelecido com a população atendida.

Nos encontros são abordados diversos assuntos levantados pelos participantes e que remetem à importância da atuação interdisciplinar, descritos a seguir:

- Rotina do CTI: procedimentos ao ingressar no setor, devido ao risco de infecção dentro de unidade fechada; dúvidas relativas ao contato físico com paciente e esclarecimento sobre a necessidade e função de drenos, sondas e outros dispositivos em uso pelo paciente;

- Referenciamento do atendimento do (a) assistente social nos andares e do (a) psicólogo(a) no ambulatório, posto que estes serviços não se fixam no CTI, ao contrário dos profissionais médico(a), enfermeiro(a) e fisioterapeuta;

- Acolhimento de sentimentos de saudade, tristeza e medo, bem como dos sentimentos negativos e elaboração dos mesmos (raiva do paciente, raiva de Deus, etc); reflexão acerca da necessidade de encontrar uma justificativa para o surgimento/agravamento da doença;

- Orientações a respeito de reclamações e elogios, principalmente através do serviço de ouvidoria do hospital;

- Esclarecimento a respeito dos procedimentos após alta do CTI;

- Visita infantil: esclarecimento quanto à abordagem a ser utilizada pela família e pelos profissionais junto à criança;

- Aspecto/suporte religioso: acolhimento das questões e indicação do Núcleo de Apoio Voluntário Espiritual (NAVE), existente no Hospital do Câncer I/INCA;

- Conflitos familiares e preocupações que repercutem na visita e no cuidado ao paciente;

- Direitos sociais do paciente oncológico, com destaque para os aspectos previdenciários e procurações, devido ao prolongado tempo de permanência no setor.

Durante a atividade, observa-se que o esclarecimento sobre a doença proporciona ao familiar/responsável a percepção do tratamento e das formas de organização a serem estabelecidas no que se refere ao processo de internação, de acordo com a evolução da doença. Com relação a esta, como já dito, não é raro nos depararmos com estágios avançados, com possibilidade imi- nente de morte, que fazem emergir nos familiares sentimentos de angústia, de receio quanto à reação do paciente ao saber do agravamento da doença, conflitos familiares e até mesmo a negação da doença.

Sem o estabelecimento de laços com a família é praticamente inviável o diálogo franco sobre as situações limite, que por sua vez exigem decisão. No momento em que os esforços terapêuticos são limitados é que é possível se ter clareza da dimensão que assume a boa relação dos profissionais para com a família. Caso contrário, a resistência e a não aceitação do agravamento do quadro clínico do paciente pode prolongar o sofrimento, sem benefícios a nenhum dos envolvidos.

Desse modo, o trabalho interdisciplinar tem como alicerce o estabelecimento do vínculo e a melhoria no acesso dos usuários aos serviços de saúde, através da humanização da assistência. Possibilita o controle social nas decisões que envolvem escolhas, propiciando condições para que os usuários exerçam sua autonomia.

\section{Considerações finais}

Embora existam muitos estudos que ressaltem os benefícios da interdisciplinaridade, é conveniente lembrar que muitos são os obstáculos ao seu exercício na prática dos serviços de saúde. Trabalhar de forma interdisciplinar exige integração com as demais áreas envolvidas, rompendo com a fragmentação da organização do trabalho, que é reconhecidamente um dos maiores entraves à realização de um trabalho em saúde de melhor qualidade, tanto para os profissionais que o realizam quanto para aqueles que são atendidos.

A complexidade do setor Saúde pressupõe envolvimento dos diversos saberes, exigindo o exercício de uma comunicação efetiva entre os profissionais no respeito ao conhecimento de cada um no processo, e entre os profissionais e os usuários, facilitando a troca de informações e o acolhimento. No presente estudo, julgamos pertinente relatarmos nossa experiência no atendimento aos familiares/responsáveis dos pacientes internados no CTI adulto enquanto iniciativa que possibilita, além do vínculo com a equipe e da ação mais resolutiva, a expressão efetiva das demandas relativas ao adoecimento e/ou iminência da morte. 


\section{Colaboradores}

LS Alcantara, JL Sant'Anna e MGN Souza participaram igualmente de todas as etapas de elaboração do artigo.

\section{Referências}

1. Brasil. Ministério da Saúde (MS). Instituto Nacional de Câncer (INCA). [Internet]. Estimativa 2012: incidência do câncer no Brasil. Rio de Janeiro: INCA; 2011 [acessado 2013 abr 2]. Disponível em: http://www.inca.gov.br/estimativa/2012/index. asp? $\mathrm{ID}=5$

2. Brasil. Ministério da Saúde (MS). Instituto Nacional de Câncer. Portaria GM/MS No 2.439 de 8 de dezembro de 2005. Institui a Política Nacional de Atenção Oncológica: promoção, prevenção, diagnóstico, tratamento, reabilitação e cuidados paliativos, a ser implantada em todas as unidades federadas, respeitadas as competências das três esferas de gestão. Diário Oficial da União 2005; 9 dez.

3. Vidal DLC. Demanda reprimida: acesso aos serviços de saúde e Serviço Social. Serv. Soc. Soc. 2008; 29(94):132-153.

4. Brasil. Ministério da Saúde (MS). Secretaria de Atenção à Saúde. Programa Nacional de Humanização da Assistência Hospitalar. Brasília: MS; 2001.

5. Correia MVC. Controle Social. Dicionário da Educação Profissional em Saúde. 2009 [acessado 2013 abr 2]. Disponível em: http://www.epsjv.fiocruz.br/ dicionario/verbetes/consoc.html

6. Pinheiro R. Cuidado em Saúde. Dicionário da Educação Profissional em Saúde. 2009. [acessado 2013 abr 2]. Disponível em: http://www.epsjv.fiocruz.br/ dicionario/verbetes/cuisau.html

7. Benevides R, Passos E. Humanização na saúde: um novo modismo? Interface (Botucatu) 2005; 9(17):389394.

8. Lino MM, Calil AM. O ensino de cuidados críticos/ intensivos na formação do enfermeiro: momento para reflexão. Rev. esc. enferm. USP 2008; 42(4):777783.

9. Brasil. Ministério da Saúde (MS). Portaria MS nº. 1071 de 4 de julho de 2005. Política Nacional de Atenção ao Paciente Crítico. Diário Oficial da União 2005; 8 jul.

10. Shimizu HE, Ciampone MHT. As representações sociais dos trabalhadores de enfermagem não enfermeiros (técnicos e auxiliares de enfermagem) sobre o trabalho em Unidade de Terapia Intensiva em um hospital-escola. Rev. esc. enferm. USP 2002; 36(2):148-155.

11. Pusch R. Humanização e integralidade1. Rev. $S B P H$ 2010; 13(2):210-216.

12. Borges ADVS, Silva EF, Toniollo PB, Mazer SM, Valle ERM, Santos MA. Percepção da morte pelo paciente oncológico ao longo do seu desenvolvimento. Psicol. estud. 2006; 11(2):361-369.

13. Kovács MJ. Morte e desenvolvimento humano. São Paulo: Casa do psicólogo; 1992.

14. Ribeiro HP. O Hospital: história e crise. São Paulo: Cortez; 1993.

15. Andrade GRB, Vaitsman J. Apoio social e redes: conectando solidariedade e saúde. Cien Saude Colet 2002; 7(4):925-934.

16. Brasil. Ministério da Saúde (MS). Carta dos Direitos dos Usuários da Saúde. [Internet] 2006 [acessado 2013 abr 14]. Disponível em: http://bvsms.saude. gov.br/bvs/publicacoes/carta_direito_usuarios_ 2ed2007.pdf 
17. Menezes RA. Difíceis Decisões: uma abordagem antropológica da Prática Médica em CTI. Physis 2000; 10(2):27-49.

18. Duarte MJO. Os cuidadores e o cotidiano do cuidado em um serviço de saúde mental. Em Pauta, $n$. 14. Rio de Janeiro: UERJ; 1999.

19. Teixeira M, Nunes S. A Interdisciplinaridade no Programa de Saúde da Família: uma Utopia? In: Bravo MIS, Vasconcelos AM, Gama AS, Monnerat G. Saúde e Serviço Social. 1a Edição. São Paulo, Rio de Janeiro: Cortez, UERJ; 2004.

20. Gomes R, Deslandes SF. Interdisciplinaridade na Saúde Pública: um campo em construção. Rev. Latino-am. Enfermagem 1994; 2(2):103-114.

Artigo apresentado em 30/04/2013

Aprovado em 22/05/2013

Versão final apresentada em 22/05/2013 